Products and Services In Practice is provided to readers using text and images from the manufacturer, supplier or distributor and does not imply endorsement by $B D J$ In Practice. Normal and prudent research should be exercised before purchase or use of any product mentioned.

Please send product and services news through to David Westgarth, BDJ In Practice via: David.Westgarth@bda.org

\section{Your team for life}

At Colosseum Dental UK, our dentists are supported by highly-talented, skilled teams to help them become the best they can be.

The rewards include:

$\rightarrow$ Exceptional clinical facilities

$\rightarrow$ Ongoing opportunities for professional development

$\rightarrow$ Quality training

$\rightarrow$ Loyal patients

$\rightarrow$ An attractive financial package.

Dentists love working for Colosseum Dental UK! The camaraderie is great and you will be part of a family that cares about your personal fulfilment as well helping you advance your career.

We have practices in locations across the UK - we are the fastest-growing dental network in Europe - and have invested millions in modernising all our workspaces.

Find your fit at Colosseum Dental UK, to deliver exceptional dentistry for years to come. For more information about Colosseum Dental, visit www. colosseumdental.co.uk/careers.

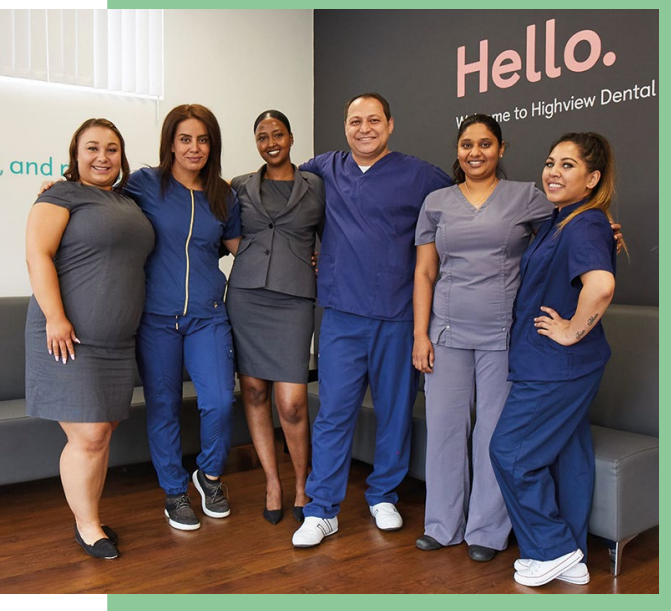

\title{
\#BrushlikeAProWithMo
}

Oral-B is on a mission to educate the UK on the importance of a good oral health routine, after research found that one in three families (33\%) in the UK are in dental crisis, following a lack of dental care in the last 12 months.

Oral-B have launched a new TV advert featuring multiple Olympic, World and European Champion Athlete and dad of four, Sir Mo Farah CBE, who knows all about the importance of keeping teeth in good condition. A few years ago Mo had a serious tooth infection which saw him admitted to hospital and miss a period of training. Mo is partnering with Oral-B to help raise awareness of the importance of good oral health and to inspire the nation to adopt better oral care routines at home.

'Good dental hygiene is crucial to everyday health', said Farah, adding: 'I'm a prime example of the effect dental issues can have on personal health and the ability to perform in your job. I urge the nation to look after theirs and their children's teeth and general oral health, just as they would other aspects of their wellbeing, to keep them away from any nasties that poor oral hygiene can lead to.

Oral-B is proud to partner with Mo Farah to improve the oral health of the nation, by inspiring people to adopt better oral care habits from an early age and to \#BrushLikeAProWithMo.

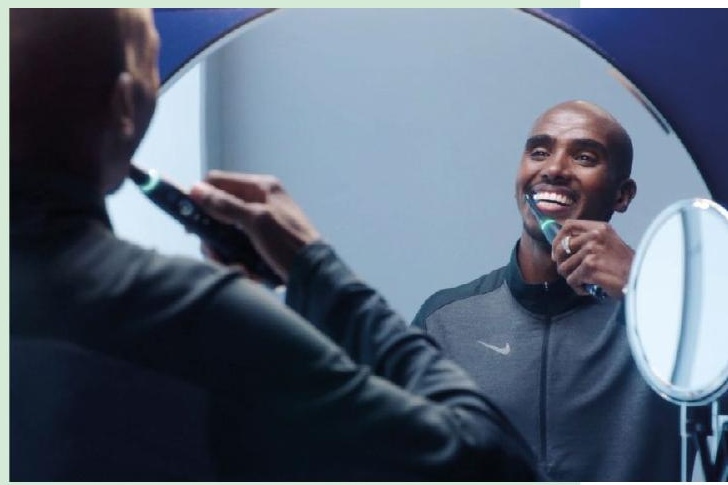

\section{Heading in the right direction}

In the dental practice, reducing waste can be hard when infection control protocols require some items to be disposed of immediately after use, or washed after one wear.

But we can still live more sustainably, by looking at the products we use and recommend.

Forest Stewardship Council (FSC) certification enables the identification of wood and paper made with materials from responsibly-managed forests. From TANDEX, the WOODI interdental brush has a handle made from FSC-certified birchwood - the box is fully recyclable too! TANDEX has long supported environmental concerns. To find out more about this, and the WOODI, visit https:// tandex.dk/.

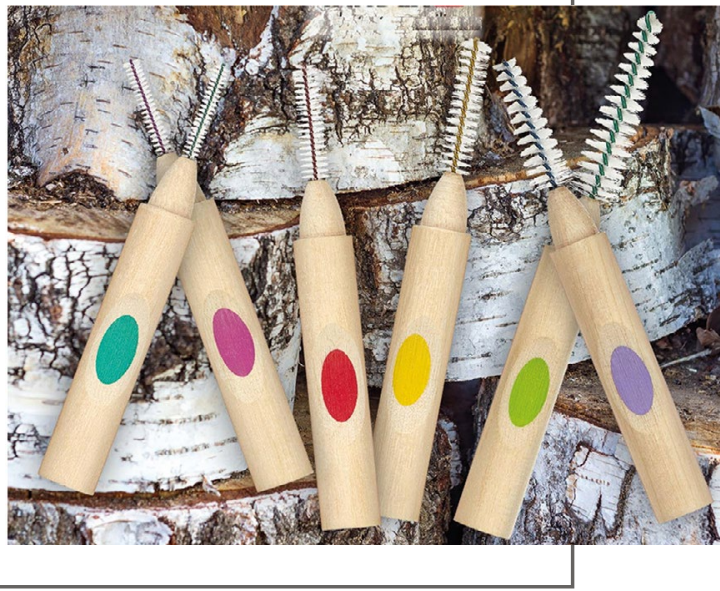

